\title{
Optimization of a bio-inspired sound localization sensor for high directional sensitivity
}

\author{
Andrew Reid \\ Centre for Ultrasonic Engineering, \\ University of Strathclyde, \\ Glasgow, UK \\ andrew.reid@strath.ac.uk
}

\author{
Deepak Uttamchandani \\ Centre for Microsystems and \\ Photonics, \\ University of Strathclyde, \\ Glasgow, UK
}

\author{
James F.C. Windmill \\ Centre for Ultrasonic Engineering, \\ University of Strathclyde \\ Glasgow, UK
}

\begin{abstract}
Miniaturization of sound localization sensors arrays is heavily constrained by the limited directional cues in intensity difference and phase difference available at the microscale. Micro-Electro Mechanical System (MEMS) sound localization sensors inspired by the auditory system of Ormia ochracea offer a potential solution to this problem by the apparent amplification of the available intensity and phase difference between the measurement points. An inherent limitation of these existing systems is that significant amplification of these cues is only available at or close to one of the resonant frequencies of the device, severely limiting it application as a directional microphone. Here we present the process of optimization of a sound localization sensor for the maximum amplification of directional cues across a narrow bandwidth, increasing the signal to noise ratio and the reading accuracy for sound localization measurements.
\end{abstract}

Keywords-Sound localization; MEMS; Ormia ochracea; bioinspired

\section{INTRODUCTION}

Miniature directional microphones have applications ranging from hearing aid systems [1] to military systems for locating the positions of snipers [2],[3],[4]. For miniaturization of such systems to be considered successful, a requirement is the use of a single sensor which has inherent directional sensitivity. Insect inspired microphones offer a solution to address this challenge, since certain insects such as Ormia ochracea are known to have auditory systems with a high directional capability [5],[6].

Ormia ochracea inspired directional microphones comprise two diaphragms which are mechanically linked, either by the addition of a lever arm attached to the center of each diaphragm [7],[8] or, more commonly, by connecting two cantilever microphones via a single, torsional central pivot [9],[10]. The connected diaphragms then have two principal mode shapes; the 'translational' mode in which the diaphragms oscillate in phase and the 'rocking' mode in which they oscillate in anti-phase. The translational mode is excited by the sum of the pressure on both the diaphragms, while the rocking mode is stimulated by the pressure difference [11]. When these modal forces are of comparable strength the motion of the diaphragm on the contralateral side of the sound source is repressed and that of the ipsilateral diaphragm is reinforced, creating a difference in power and phase of oscillation between the diaphragms which is many times greater than that of the stimulating sound wave. These quantities are styled the mechanical interaural intensity difference (mIID) and the mechanical interaural phase difference (mIPD).

The amplification of directional cues depends on the modes being of comparable strength and as such the useful range in which amplification can occur is constrained to be near one of the resonance modes [12]. While the bandwidth of the resonance modes can be increased with an increase in the damping of the system, thereby increasing the range in which amplification of directional cues may take place, higher damping in MEMS is often the result of thin film damping which varies considerably between the rocking and translational modes [13] and severely compromises the mechanical sensitivity of the system to sound pressure. Current iterations of MEMS directional microphones resolve this problem by using relatively high $\mathrm{Q}$ systems and optimizing their operation for a limited amplification of intensity and phase difference that is stable across a wide frequency range [14],[15].

In counter-sniper detection systems, as well as unmanned aerial vehicle (UAV) detection systems the frequency spectrum of the sound source is often well known in advance [4], allowing a sound localization system to be tailored to frequencies at which the spectral signature is known to have consistent energy. If the requirement for wide bandwidth operation is removed, some freedom is granted to increase the magnification of directional cues to create an accurate, single purpose sound localization sensor. Accurate measurement of the angle to the sound source depends on the rate of change of MIID and MIPD to sound source angle, the directional sensitivity, and the linearity of that directional sensitivity. The primary determinant of this is the coupling strength between the membranes, the stiffness of the mechanical connection between them, which must be tailored to the damping conditions of the system [16]. For any given damping regime there exists a point of 'dual optimization' in which the rate of change of mIPD or mIID reaches a local maximum and the deviation from a linear relationship between mIPD and mIID is at a minimum within $30^{\circ}$ of the device midline [17].

At this point of dual optimization the directional sensitivity for a separation of $2 \mathrm{~mm}$ between the diaphragms is $1.5-1.8$ degrees phase difference per degree change in angular azimuth and $1.5-1.8 \mathrm{~dB}$ per degree azimuthal angle for the mIPD and mIID measures respectively, a figure which remains constant regardless of the angular range of operation or damping conditions. Further amplification of the directional cues therefore comes at the cost of the linear sensitivity of the measurement. In addition the mIID measure, as a ratio of the 
amplitudes of the diaphragms of the device, introduces a Cauchy-like noise further reducing the accuracy of sound localization measurements.

In this study we investigate deviations from the dual optimization position in the low damping conditions available on a single layer silicon on insulator (SOI) MEMS device. In these conditions the strength of the coupling between the membranes may be increased beyond the dual optimization point without significant loss of linearity of sensitivity. The resultant increase in signal significantly improved the signal to noise ratio of the mIID measure, allowing both the MIPD and mIID measures to be used on a single device. The device presented here demonstrates a directional sensitivity in the mIID measure of $1.6 \mathrm{~dB}$ per degree and in the mIPD measure of 8 degrees phase difference per degree change in azimuthal angle, a seven fold increase. The average error in returned sound source angle using the mIPD measure was $0.24^{\circ}$ and using the mIID measure was $0.44^{\circ}$.

\section{THEORY AND DESIGN}

\section{A. Lumped parameter model}

$O$. ochracea inspired directional microphones can be modeled with each diaphragm being treated as a mass-springdamper system coupled by a spring and dashpot pair. The resulting system of equations can then be decomposed into the individual mode shapes, allowing the amplitude of displacement of each of the diaphragms to be described as a superposition of the rocking and translational modes.

$$
x=\frac{p_{0} s}{k}\left(\frac{\cos (\phi / 2)}{\eta^{2}-\Omega^{2}+\imath 2 \Omega \eta \xi_{t}} \pm \imath \frac{\sin (\phi / 2)}{1-\Omega^{2}+\imath 2 \Omega \xi_{r}}\right)
$$

Here $s$ is the surface area of a diaphragm, $p_{0}$ is the amplitude of the incident pressure wave, $\Omega$ is the frequency normalized to the resonance mode frequency, $\eta$ is the ratio of the translational mode frequency to the rocking mode frequency and $\xi_{\mathrm{t}}$ and $\xi_{\mathrm{r}}$ are the damping coefficients associated with the resonance and translational modes respectively. The phase difference in the acoustic wave, $\varphi$, is given by:

$$
\varphi=2 \pi f d \sin (\theta) / v
$$

where $f$ is the frequency of the sound wave, $v$ is the speed of sound, $d$ is the distance between the measurement points and $\theta$ is the azimuthal angle of the sound waOptimizve. The mIID and mIPD can then be expressed as a ratio of the modal forces:

$$
\begin{aligned}
& m I I D=20 \log \left|\frac{\Gamma+\imath \tan (\phi / 2)}{\Gamma-\imath \tan (\phi / 2)}\right| \\
& m I P D=\angle \frac{\Gamma+\imath \tan (\phi / 2)}{\Gamma-\imath \tan (\phi / 2)}
\end{aligned}
$$

where:

$$
\Gamma=\frac{1-\Omega^{2}+\imath 2 \Omega \xi_{r}}{\eta^{2}-\Omega^{2}+\imath 2 \Omega \eta \xi_{t}}
$$

The mIID measure is notably a ratio of the displacements of the diaphragms, both of which could be considered to have some additive noise. Off the resonance peaks, where the signal is small compared to the noise, the ratio of the two displacements shows a characteristic Cauchy distribution.

Two further measures are useful in assessing the accuracy of sound source measurement: the average directional sensitivity (ADS) over the angular range of operation and the non-linearity (NL), measured as the deviation of the MIID or mIPD from the ideal linear sensitivity given by the product of ADS and azimuthal angle (a more complete explanation of these measures is given in the supplementary materials for [17]).

\section{B. Estimation of damping parameters}

The directional sensitivity and angular range of sound localization is extremely sensitive to changes in damping, making a good estimate of the $\mathrm{Q}$ of the diaphragm an essential prerequisite to design. As the device contains no back plate, the principle contributors to damping are expected to be the viscous and thermal losses in the boundary layer around the device. The depths of the boundary layers are given by:

$$
\begin{aligned}
& \delta_{v}=\sqrt{\frac{2 \mu}{\omega \rho_{0}}} \\
& \delta_{t}=\sqrt{\frac{2 k}{\omega \rho_{0} C_{p}}}
\end{aligned}
$$

In order to obtain a single lumped damping coefficient for each of the mode shapes estimations of the total damping force are made via Finite Element Analysis using COMSOL Multiphysics' thermoacoustic module. The model comprises three components: the diaphragm, modeled as a shell layer made of single crystal silicon, the air domain around the diaphragm, modeled using the thermoacoustics interface, and an extended air domain, modeled using the simpler pressure acoustics interface. A plane sound wave with an amplitude of $1 \mathrm{~Pa}(94 \mathrm{~dB}$ ref $20 \mu \mathrm{Pa})$, is simulated with the direction of propagation perpendicular to the face of the diaphragms (Figure 1).

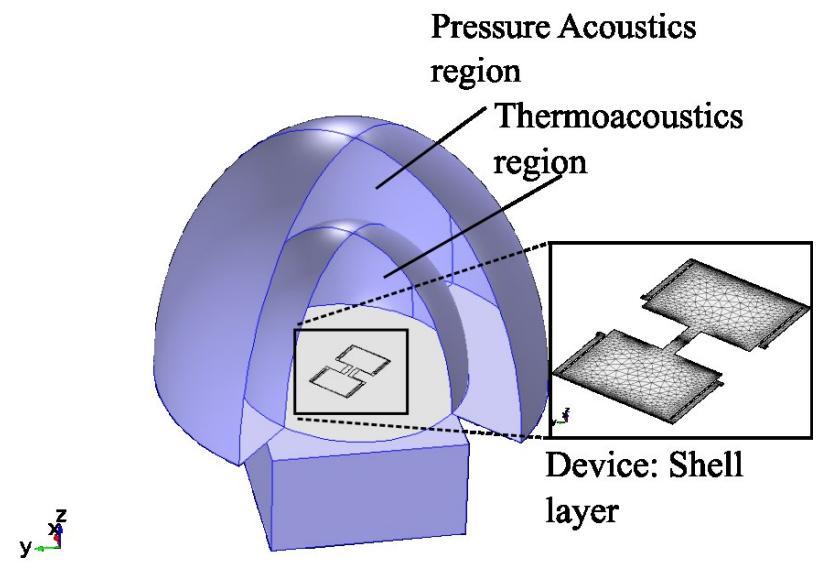

Figure 1: The COMSOL model air domain is split, with viscosity and thermal conductivity omitted in the outer layer (Pressure Acoustics domain) and the more computationally intensive thermoacoustic equations solved in the smaller inner layer. The device is a shell layer 10 $\mu m$ thick attached to the substrate at the pivots and the end of each of the spring arms, simulating the piezoelectric sensing device. A second model with the spring arms omitted was also generated simulating the capacitive comb sensing device. 
TABLE I. SIMULATED RESONANCE FREQUENCIES AND DAMPING

\begin{tabular}{|c|c|c|c|c|}
\hline \multirow{2}{*}{$\begin{array}{c}\text { Air } \\
\text { temp. }\end{array}$} & \multicolumn{4}{|c|}{ COMSOL simulated resonances and damping } \\
\cline { 2 - 5 } & \multicolumn{2}{|c|}{ Resonance Frequencies $(\mathrm{Hz})$} & \multicolumn{1}{c|}{ Damping Coefficients } \\
\cline { 2 - 5 } & Rocking & Translational & Rocking & Translational \\
\hline $0^{\circ}$ & 8224.5 & 8846 & $4.43 \times 10^{-3}$ & $5.52 \times 10^{-3}$ \\
\hline $20^{\circ}$ & 8229 & 8851.4 & $5.25 \times 10^{-3}$ & $5.25 \times 10^{-3}$ \\
\hline $20^{\circ}$ & 3165.8 & 3403.2 & $40.2 \times 10^{-3}$ & $41.3 \times 10^{-3}$ \\
\hline
\end{tabular}

The simulated resonance frequencies and damping coefficients (Table 1) show Q factors in the region of $95-115$ for the higher frequency, piezoelectric sensing devices $10-12$ for the lower frequency, capacitive comb sensing devices. The change in damping with temperature can also have a significant effect on the accuracy of the device. Between $0^{\circ} \mathrm{C}$ and $20^{\circ} \mathrm{C}$ the directional sensitivity around the midline as a result of an $18 \%$ change in the damping of the rocking mode will change the directional sensitivity around the midline by $16 \%$ (Figure 2) leading to a potential error in the measurement of sound source angle of $1.4^{\circ}$ at the extremes of the linearly sensitive range.

\section{Dual optimization and 'over-coupling'}

The frequency at which maximum amplification of both mIID and mIPD occurs in lightly damped systems is typically on or near that of the rocking mode resonance frequency due to the much higher modal weight of the translational mode. For any given damping conditions the point of dual optimization may then be found parametrically by altering the coupling strength between the diaphragms. The point of dual optimization is recognized by a local maxima in ADS collocated in the frequency domain with a local minima in nonlinearity.

The directional sensitivity at the point of dual optimization in the MIPD measure is consistently seven times the phase difference of the stimulating sound wave between the two diaphragms while the non-linearity falls within $0.02-0.05$ degrees within $30^{\circ}$ of the midline. Reducing the angular range of the device does not afford any improvement in sensitivity at this dual optimization point. However by reducing the angular range in the case of very light damping, the loss of accuracy from non-linear sensitivity from over-coupling beyond the dual optimization point can be very slight, moving from an ideal 0.005 degrees to $0.01-0.02$ degrees which remains a significant improvement on devices with a larger angular range of operation (Figure 3). By increasing the signal in this way it is hoped to improve the signal to noise ratio of the MIPD and mIID and therefore increase the accuracy of angular measurement.

\section{EXPERIMENTAL RESULTS}

The directional response of the completed device was measured using a scanning laser vibrometer (Polytec, PSV-300F) using and OFV056 scanning head fitted with close up attachment. The device was fixed vertically with laser Doppler measurements being taken on the rear face (through the trench

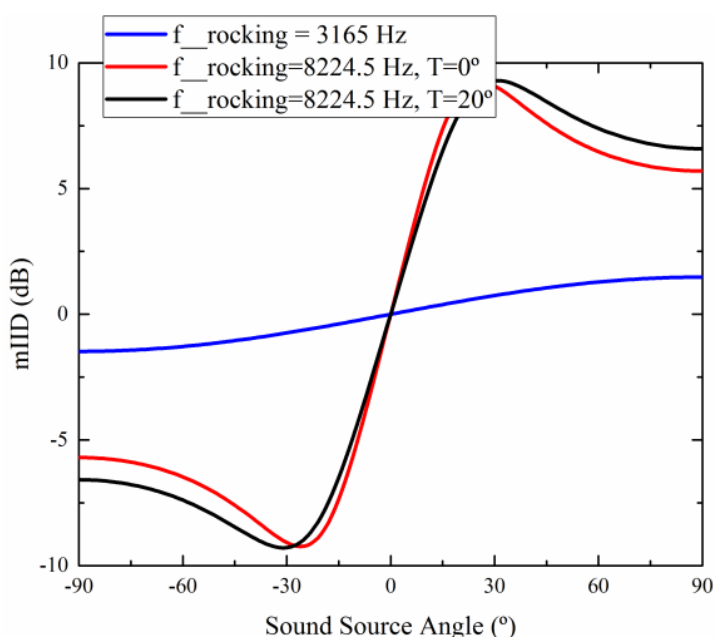

Figure 2: The effect of damping on the sensitivity of the device is pronounced. Each of the three cases above have the same coupling strength, however the lower resonance frequency device $Q$ is much reduced giving a sensitivity around the midline of $0.025 \mathrm{~dB} /{ }^{\circ}$ (and 0.2 degrees phase difference per degree change in sound source angle in the $m I P D$ measure). In the same device the temperature change from $0^{\circ} \mathrm{C}-$ $20^{\circ} \mathrm{C}$ can result in a change of sensitivity from $0.52 \mathrm{~dB} /{ }^{\circ}$ to $0.45 \mathrm{~dB} /{ }^{\circ}$ around the midline $\left(3.03^{\circ}\right.$ phase $/{ }^{\circ}$ azimuth to $2.516^{\circ}$ phase $/{ }^{\circ}$ azimuth in the mIPD measure).

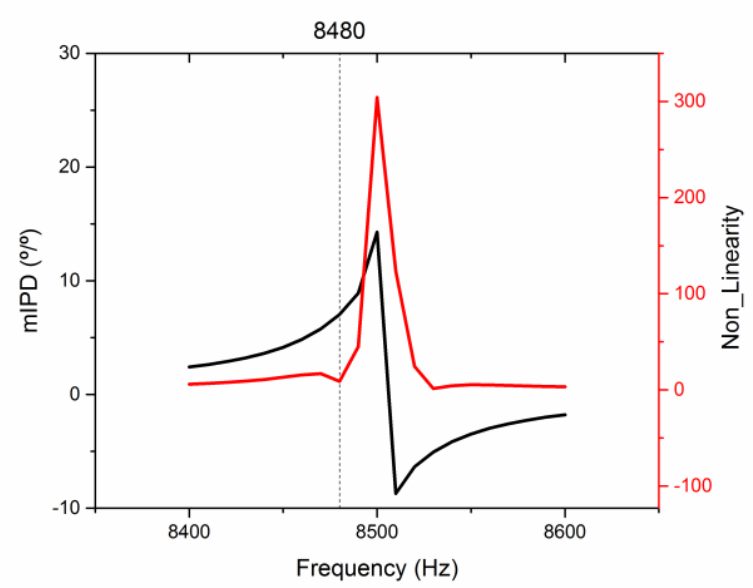

Figure 3: Theoretical average directional sensitivity and non-linearity in an 'over-coupled' device. Here it is the maximum non-linearity which is aligned with the maximum directional sensitivity, as opposed to the 'dual optimized' case where the minima in non-linearity and the maximum directional sensitivity are co-located. The increase in directional sensitivity from over-coupling allows operation away from the maximum while still showing an improvement over the sensitivity of the dual optimized design with only a slight increase in non-linearity. The spike in non-linearity manifests as a null in directional sensitivity within $1^{\circ}-2^{\circ}$ of the midline.

layer) and the sound source presented to the top layer of the device moving in $10^{\circ}$ increments around the axis of the pivots from $-90^{\circ}$ to $90^{\circ}$ with measurements at $1^{\circ}$ intervals being taken within $10^{\circ}$ of the midline.

The measured resonance frequencies were $8560 \mathrm{~Hz}$ for the rocking mode and $9281 \mathrm{~Hz}$ for the translational mode, with the peak mIID being found at $8572 \mathrm{~Hz}$ and the peak mIPD at 8565 Hz. Damping coefficients were measured from the half power 

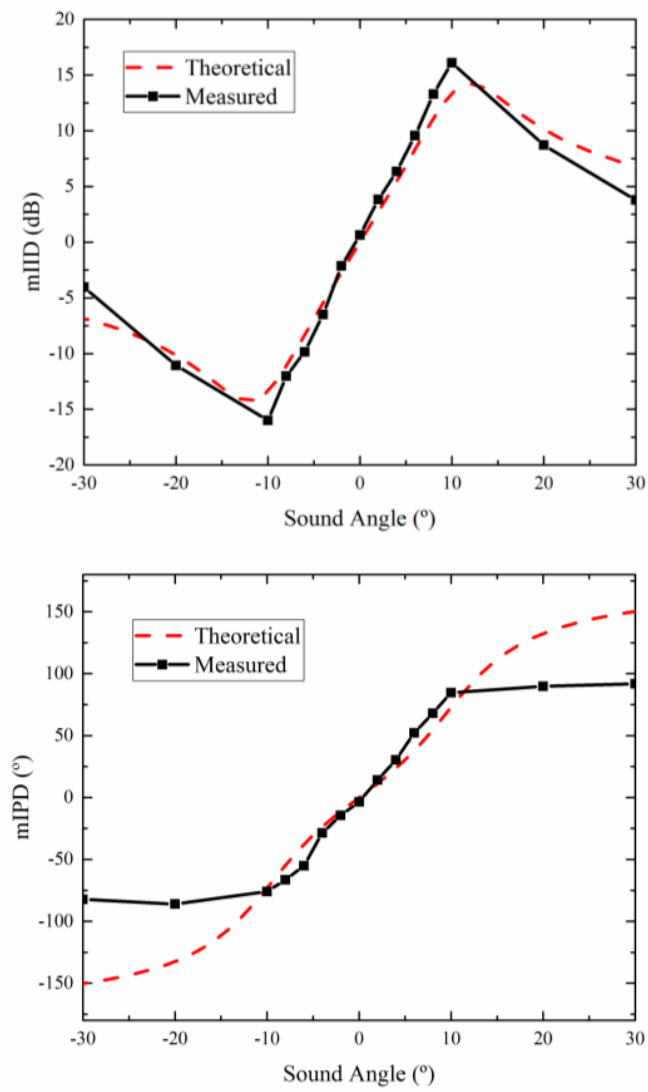

Figure 4: Comparison of lumped parameter model estimates for mIID and measured values in the Piezoelectric device based on the measured device frequencies of $8560 \mathrm{~Hz}$ and $9281 \mathrm{~Hz}$ and the damping parameters of 0.004 in the translational mode and 0.003 in the rocking mode. mIPD measurements of directional sensitivity agreed well with those modeled. however the measured signal was found to saturate before predicted.

bandwidth of each of the resonance mode peaks at 0.005 for the translational mode and 0.003 for the rocking mode. The mIID and mIPD measurements were taken at $8540 \mathrm{~Hz}$, which was found to produce the largest directional sensitivity without loss of linearity near $0^{\circ}$ sound source angle (Figure 4). Peak values were attained at $10^{\circ}$ for each with an average directional sensitivity of $1.6 \mathrm{~dB}$ per degree in $\mathrm{mIID}$ and of $8^{\circ}$ phase difference per degree sound source incidence angle in mIPD. Mean absolute error was $0.38 \mathrm{~dB}$ in mIID and $3.49^{\circ}$ in mIPD for a sound source angle measurement error of $0.24^{\circ}$ and $0.44^{\circ}$ respectively.

The scaling parameter of the Cauchy noise was estimated at $0.52 \mathrm{~dB}$ by taking half the interquartile range from measurements made with the sound source at $0^{\circ}$ (wave fronts parallel to the top layer of the device). The error of the device could therefore be far larger, but would be most likely to fall within $0.325^{\circ}$ of the sound source angle.

\section{CONCLUSION}

The methods described here show the design process behind an Ormia ochracea inspired sound localization sensor with a directional sensitivity many times great than that of a 'dual optimized' design. In accepting the limitations on bandwidth and angular range of operation a single purpose sound localization sensor can be created. While the single device presented here is capable of sound localization to an accuracy of less than half a degree, FEA simulations of the change in directional sensitivity with relatively small changes in ambient pressure or temperature would preclude a single, such device being used outside laboratory conditions. Given the limited angular range of operation the likely end use of these devices would be as part of an array, allowing some compensation for poor calibration of the sensor to be performed.

\section{ACKNOWLEDGMENT}

The research leading to these results has been supported by the DGA-DSTL UK French PhD program.

\section{REFERENCES}

[1] R. Miles and R. Hoy, "The development of a biologically-inspired directional microphone for hearing aids," Audiol. Neurotol., vol. 11, no. 2, pp. 86-96, 2006.

[2] J. Chang, W. Mendyk, L. Thier, P. Yun, A. LaRow, S. Shaw, and W. Schoenborn, "Early attack reaction sensor (EARS), a man-wearable gunshot detection system,” Proc. SPIE, vol. 6201. p. 62011T-62011T-8, 2006.

[3] T. Damarla, L. Kaplan, and G. Whipps, "Sniper localization using acoustic asynchronous sensors," Sensors Journal, IEEE, vol. 10, no. 9, pp. 1469-1478, 2010.

[4] H. Liu, L. Currano, D. Gee, B. Yang, and M. Yu, "Fly-ear Inspired Acoustic Sensors for Gunshot Localization," in Proceedings of SPIE, 2009, vol. 7321, p. 73210A-73210A-10.

[5] R. N. Miles, D. Robert, and R. R. Hoy, "Mechanically coupled ears for directional hearing in the parasitoid fly Ormia ochracea.," J. Acoust. Soc. Am. vol. 98, no. 6, pp. 3059-70, Dec. 1995.

[6] D. Robert, R. N. Miles, and R. R. Hoy, "Directional hearing by mechanical coupling in the parasitoid fly Ormia ochracea.," J. Comp. Physiol. A., vol. 179, no. 1, pp. 29-44, Jan. 1996

[7] L. J. Currano, H. Liu, D. Gee, B. Yang, and M. Yu, "Microscale implementation of a bio-inspired acoustic localization device," Proc. SPIE, vol. 7321, no. 301, p. 73210B-73210B-8, May 2009.

[8] H. Liu, M. Yu, L. Currano, and D. Gee, "Fly-Ear Inspired Miniature Directional Microphones: Modeling and Experimental Study," JASA, vol. 129, no. 5, pp. 1-7, 2009.

[9] W. Cui, R. Miles, and Q. Su, "A Robust Miniature Silicon Microphone Diaphragm,” IFSA, Sensors Transducers J. ( ..., vol. 7, no. October, pp. 63-77, 2009.

[10] Q. Su, R. Miles, and M. Weinstein, "Response of a biologically inspired MEMS differential microphone diaphragm," in Proceedings of the SPIE AeroSense, 2002

[11] D. Robert, R. N. Miles, and R. R. Hoy, "Tympanal mechanics in the parasitoid fly Ormia ochracea: intertympanal coupling during mechanica vibration," J. Comp. Physiol. A Sensory, Neural, Behav. Physiol., vol. 183, no. 4, pp. 443-452, Oct. 1998.

[12] H. Liu and M. Yu, "Fly-Ear Inspired Miniature Acoustic Sensors," smc.hit.edu.cn, 2011.

[13] K. Chang, S. Lee, and S. Li, "Squeeze film damping effect on a MEMS torsion mirror," J. Micromechanics ..., vol. 556, no. 12, pp. 556-561, 2002.

[14] R. N. Miles, Q. Su, W. Cui, M. Shetye, F. L. Degertekin, B. Bicen, C. Garcia, S. Jones, and N. Hall, "A low-noise differential microphone inspired by the ears of the parasitoid fly Ormia ochracea.," J. Acoust. Soc. Am., vol. 125, no. 4, pp. 2013-26, Apr. 2009.

[15] W. Cui, R. Miles, Q. Su, and D. Homentcovsci, "A Bio-Inspired Miniature Comb Sense Differential Microphone Diaphragm," in Proceedings of the 2010 ASME International Mechanical Engineering Congress \& exposition, 2010, vol. 1 , no. 607 , pp. $1-6$

[16] A. Lisiewski, "Fly-Ear Inspired Miniature Acoustic Sensors," 2011.

[17] H. Liu, L. Currano, D. Gee, T. Helms, and M. Yu, "Understanding and mimicking the dual optimality of the fly ear.," Sci. Rep., vol. 3, p. 2489, Jan. 2013 